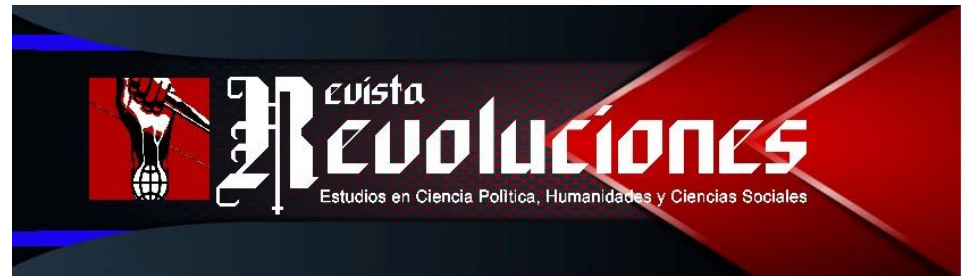

\title{
PROGRAMA NACIONAL DE ASISTENCIA SOLIDARIA PENSIÓN 65 Y EL BIENESTAR SOCIAL DE LOS BENEFICIARIOS EN LOS DISTRITOS DE PUNO Y CHUCUITO
}

\section{National program of solidarity assistance Pension 65 and welfare social of beneficiaries in the districts of Puno and Chucuito}

\author{
Humberto Tintaya \\ Duran' \\ UNIVERSIDAD NACIONAL DEL \\ ALTIPLANO \\ PERÚ \\ tintaya1001@gmail.com \\ https://orcid.org/oooo-ooo1- \\ 7296-4106
}

\author{
Richard Sullca Cáceres 2 \\ UNIVERSIDAD NACIONAL DEL \\ ALTIPLANO \\ PERÚ \\ sullcacaceresr@gmail.com \\ https://orcid.org/o0oo-0002- \\ 8853-9179
}

DOI: https://doi.org/10.35622/j.rr.2019.01.005

Recibido: 03-I-2019 / Aceptado: 27-I-2019 / Actualizado: 01-VI-2020

\section{Resumen}

La investigación analiza la relación que existe entre las líneas de intervención del Programa Nacional de Asistencia Solidaria Pensión 65 y el bienestar social de los beneficiarios en los distritos de Puno y Chucuito. se realizó un estudio cuantitativo, no experimental de carácter descriptivo y correlacional. El interés investigativo se centra en analizar la relación entre la subvención económica, acceso a servicios públicos y la intervención de saberes productivos con el bienestar social de los adultos mayores. Estas dimensiones se analizaron mediante el uso de las encuestas y para el análisis de resultados se utilizó el software estadístico SPSS; para evaluar la significancia se recurrió a la prueba Chi cuadrado. Gracias a ello se determinó que la subvención económica que otorga el programa no tiene una relación significativa con el bienestar social de los beneficiarios, por ser limitado y mayormente destinado para cubrir gastos de alimentación. El acceso a servicios públicos es independiente y no tiene relación significativa con el bienestar social, debido a que la mayor parte de beneficiarios necesita de atención médica por tener algún tipo de enfermedad y con una ponderación alta en el distrito de Chucuito no tienen acceso de manera oportuna a los servicios de salud. Finalmente se

\footnotetext{
${ }^{1}$ Licenciado en Sociología por la Facultad de Ciencias Sociales, Universidad Nacional del Altiplano Puno - Perú. Con conocimientos en Gestión Pública y Gestión de Programas Sociales. Con experiencia en Gestión Pública y administración Pública.

${ }^{2}$ Licenciado en Sociología por la Facultad de Ciencias Sociales, Universidad Nacional del Altiplano Puno - Perú. Y Abogado de la Universidad Andina Néstor Cáceres Velásquez Juliaca - Perú. Con conocimientos en Gestión Pública, Derecho Administrativo. Con experiencia en Gestión Pública.
} 
concluye en que existe una relación entre la intervención de saberes productivos y bienestar social de los beneficiarios del Programa Pensión 65.

Palabras Clave: Programa Pensión 65, bienestar social, saberes productivos, servicios públicos, subvención económica.

\section{Abstract}

The research analyzes the relationship between the lines of intervention of the National Solidarity Assistance Program Pensión 65 and the social welfare of the beneficiaries in the districts of Puno and Chucuito. A quantitative, non-experimental, descriptive and correlational study was carried out. The research interest is focused on analyzing the relationship between economic subsidy, access to public services and the intervention of productive knowledge with the social well-being of the elderly. These dimensions were analyzed through the use of the surveys and for the analysis of results the statistical software SPSS was used; To assess the significance, the Chi-square test was used. Thanks to this, it was determined that the economic subsidy granted by the program does not have a significant relationship with the social welfare of the beneficiaries, as it is limited and mostly intended to cover food expenses. Access to public services is independent and does not have a significant relationship with social welfare, because most beneficiaries need medical attention because they have some type of illness and with a high weighting in the district of Chucuito they do not have access Timely to health services. Finally, it is concluded that there is a relationship between the intervention of productive knowledge and social welfare of the beneficiaries of the Pension 65 Program.

Keyword: Pension 65 Program, social welfare, productive knowledge, public services, economic subsidy.

\section{INTRODUCCIÓN}

El tema del envejecimiento en el mundo ha formado parte de la agenda de los gobiernos desde mediados del siglo pasado. Es así que, en 1948 la Asamblea General de las Naciones Unidas, aprobó la Resolución 213 sobre el proyecto de declaración de los derechos de la vejez. Posteriormente, en 1977, se plantea la necesidad de organizar una asamblea mundial sobre las personas de edad. En esa perspectiva, en 1982, con la realización de la Asamblea Mundial sobre el Envejecimiento en Viena, Austria, en 1982, se adopta el Plan de Acción Internacional de Viena sobre el envejecimiento, en el que se encuentra inmerso el Perú. Este Plan recomienda acciones en el ámbito del empleo y la seguridad económica, la salud y la nutrición, la vivienda, la educación y el bienestar social (CEPAL, CELADE, 2007).

Sin embargo, una de las problemáticas que más a menudo se presenta en los adultos mayores, está referido a los problemas con la salud, esto produce una disminución en la capacidad funcional de los adultos mayores, y generalmente no tienen acceso a servicios de salud de calidad, en ese contexto la Organización Mundial de la Salud (OMS, 2015) menciona que "en los países de ingresos bajos o en entornos de escasos recursos de todo el mundo, el acceso a servicios de salud suele ser limitado" (p.6). Esto lo podemos evidenciar en el estudio de la 
OMS (2015) sobre envejecimiento y salud de los adultos mayores en el mundo; según el estudio la cobertura de salud efectiva en los países analizados iba desde el $21 \%$ de los pacientes en México al 48 \% en Sudáfrica, lo que indica que gran parte de los adultos mayores se privan de servicios de salud, los subutilizan, o por último terminan empobrecidos al tener que pagar por servicios de salud. Por otro lado, las limitaciones físicas en la vejez se asocian a las condiciones de vulnerabilidad económica, generando cierto grado de pobreza (Salvador, 2015).

En el Perú, los adultos mayores afrontan problemáticas como el analfabetismo, exclusión social, pobreza y abandono familiar principalmente; según el Instituto Nacional de Estadística e Informática (INEI, 2018) el 17.4\% de la población de 60 y más años de edad no sabe leer ni escribir, aunado a esto, el $38.4 \%$ de adultos mayores de 70 y más años viven solos. Asimismo, según los datos del Censo Nacional 2017, la población de adultos mayores está incrementando, ya que representan el $11.9 \%$ de la población total del país, y con ello, las necesidades y problemáticas de los adultos mayores irán incrementándose; es posible que las políticas que se vienen ejecutando no puedan cubrir en cobertura al total de adultos mayores.

A nivel local, el problema de analfabetismo sigue siendo alarmante en los adultos mayores de 65 y más años; es así, que en el distrito de Puno el $21.2 \%$ de personas de 65 y más años no saben leer ni escribir, además que el $17.9 \%$ son mujeres. Por otro lado, en el distrito de Chucuito, el $38 \%$ de personas de 65 y más años tampoco saben leer ni escribir, de las cuales el 30.6\% la conforman las mujeres. Además, en relación al abandono familiar, el distrito de Puno es uno de los 10 distritos de la región, con el mayor número de adultos mayores que viven solos, esto demuestra que existe mayor cantidad de adultos mayores en situación de abandono en el distrito de Puno (INEI, 2018).

Por lo anterior, las problemáticas anteriores se han presentado desde antes, y el Estado ha ido formulando políticas públicas para afrontarlas, en ese contexto se crea el Programa Nacional de Asistencia Solidaria Pensión 65, con el objetivo de contribuir al bienestar de la población adulta a través del otorgamiento de una pensión no contributiva, y de manera concreta el programa pretende reducir la pobreza extrema en adultos mayores del Perú. Sin embargo, dicha subvención económica de doscientos cincuenta es limitado, por lo que sólo alivia temporalmente la pobreza extrema en adultos mayores, y todas las problemáticas mencionadas anteriormente.

En este sentido, en los distritos de Puno y Chucuito, existen beneficiarios del Programa Pensión 65, muchos de los cuales generalmente viven solos, y sólo cuentan con la subvención otorgada por el programa para cubrir sus necesidades básicas. En ese contexto, nuestra investigación se motiva en saber si las líneas de intervención del programa contribuyen con el bienestar social de los adultos mayores en ambos distritos. Además, con la investigación se pretende conocer desde un punto de vista objetivo, la realidad de los adultos mayores, partiendo de rubros como el acceso a servicios básicos, condición física y desarrollo personal de los adultos mayores. 
Para nuestra investigación, se tiene como objetivos: explicar la relación que existe entre la subvención económica y el bienestar social de los beneficiarios, precisar la relación que existe entre el acceso a los servicios públicos y el bienestar social, e identificar la relación que existe entre la intervención de saberes productivos y el bienestar social de los beneficiarios del Programa Pensión 65 en los distritos de Puno y Chucuito.

\section{METODOLOGÍA}

Se realizó un estudio con enfoque cuantitativo, de tipo descriptivo y correlacional. La muestra estuvo conformada por 286 beneficiarios de los distritos de Puno y Chucuito, de los cuales 176 pertenecían al distrito de Puno y 110 al distrito de Chucuito. Se utilizaron técnicas e instrumentos de recolección de datos, que fueron la encuesta y el cuestionario, y para el procesamiento y análisis de la información, se realizó en el software estadístico SPSS, y la prueba estadística que re realizó para evaluar la relación entre las líneas de intervención del Programa Pensión 65 y el bienestar social de los adultos mayores fue la Chi cuadrada.

Por otro lado, el nivel de análisis de la investigación es de horizonte temporal sincrónico, porque se han utilizado datos obtenidos de la realidad del año 2019, nuestra unidad de análisis estuvo conformada por los beneficiarios del Programa Pensión 65 residentes en los distritos de Puno y Chucuito; y la unidad de observación fue conformada por los directivos y representantes de los puntos focales identificados.

\section{RESULTADOS Y DISCUSIÓN}

\section{Datos demográficos de los beneficiarios del Programa Pensión 65}

Los adultos mayores que participaron en este estudio se caracterizan por tener una edad promedio de 75 a 79 años (35.5\%) en el distrito de Puno, y de 65 a 69 (46.4\%) años en el distrito de Chucuito. Respecto al género el 65.9\% corresponden al género femenino en el distrito de Puno y con un 51.8\% prevalece el género masculino en el distrito de Chucuito. El 47.7\% eran viudos en Puno y el 60.9\% eran casados en Chucuito; el 68.8\% reside en la zona urbana en el distrito de Puno y el 61.8\% de Chucuito residían en la zona rural; el 55.1\% no tiene grado de instrucción en Puno y el 32.7\% no tiene grado de instrucción en Chucuito. Y respecto a la lengua materna, el $65.5 \%$ de encuestados del distrito de Puno tienen como lengua materna al quechua y el $98.2 \%$ del distrito de Chucuito tienen como lengua materna el idioma aimara.

\section{Subvención económica y bienestar social}

En cuanto a la seguridad económica con el dinero otorgado por el Programa Pensión 65, el 96.5\% de ambos distritos si siente satisfacción, además, dicha subvención contribuye a la reducción de la pobreza extrema; al respecto, González y Julca (2018) mencionan que el 
programa contribuye a la reducción de la pobreza extrema, y Tuesta (2018) menciona que "antes de la implementación del Programa Pensión 65 los adultos mayores en el periodo 2011, no contaban una estabilidad económica, pues no podían solventar sus gastos y satisfacer sus necesidades básicas, además no contaban con una vivienda en buenas condiciones" (p.42). por otro lado, sólo el 1.7\% de beneficiarios del distrito de Puno tiene otra fuente de ingresos y el 17.3\% de beneficiarios del distrito de Chucuito tienen otra fuente de ingresos. Lo que nos hace inferir que mayoritariamente los adultos mayores forman parte de la población económicamente no activa.

Respecto a las actividades económicas que realizan, el 81.3\% de beneficiarios del distrito de Puno no realizan ningún tipo de actividad económica; sin embargo, en el distrito de Chucuito el $38.2 \%$ se dedican a la agricultura, pero esta actividad productiva que desarrollan es destinada para el consumo familiar, en otras palabras, es una producción de subsistencia, orientada a cubrir las necesidades del hogar (Elwert y Wong, 1979 en Blum, 1995). Asimismo, nuestros resultados tienen concordancia con los resultados obtenidos por Ambrosio et al. (2017) quiénes muestran que la asignación económica del Programa Pensión 65 constituye el principal ingreso de la población beneficiaria en el distrito de Huácar, Huánuco con un 46\%.

Por otra parte, en relación al monto de dinero destinado para la alimentación, se tiene a un 44.8\% del total de encuestados en ambos distritos que destinan dinero para cubrir su alimentación en un promedio de S/ 101.00 a S/ 150.00 soles; por otra parte, sólo el 0.7\% del total no destina dinero para su alimentación, siendo una proporción mínima respecto al total de encuestados.

El acceso a servicios básicos por parte de los beneficiarios del Programa Pensión 65 en los distritos de Puno y Chucuito, es otro de los aspectos tomados en cuenta, para saber si la subvención económica influye en el bienestar social, en donde el 81.3\% de los beneficiarios del distrito de Puno tienen acceso a luz y agua, frente a un $66.4 \%$ de beneficiarios del distrito de Chucuito que también tienen acceso a servicios de agua y luz; entonces de manera general el 75.5\% de beneficiarios encuestados cuenta con servicios básicos de luz y agua, sólo el 18.9\% accede a servicios de luz, y el $4.9 \%$ no cuenta con servicios básicos en ambos distritos. En relación a la tenencia de servicios higiénicos, el $68.8 \%$ de encuestados del distrito de Puno mencionan tener servicios higiénicos con red pública, frente a un 7.3\% de beneficiarios del distrito de Chucuito que también cuentan con servicios higiénicos con red pública. Por otro lado, en el distrito de Chucuito el $76.4 \%$ cuenta con letrina; sin embargo, el $17.8 \%$ de beneficiarios a nivel de los dos distritos no cuentan con servicios higiénicos; véase (Tabla 1).

Tabla 1. Acceso a servicios básicos por parte de los beneficiarios del Programa Pensión 65 en los distritos de Puno y Chucuito, 2019

\begin{tabular}{|c|c|c|c|c|}
\hline \multirow{2}{*}{ Servicios básicos } & & \multicolumn{2}{|c|}{ Distrito } & \multirow{2}{*}{-Total } \\
\hline & & $\overline{\text { Puno }}$ & Chucuito & \\
\hline & $\mathrm{N}^{\circ}$ & 28 & 26 & \\
\hline LuZ & $\%$ & $15,9 \%$ & $23,6 \%$ & $18,9 \%$ \\
\hline Agua & $\mathrm{N}^{\circ}$ & 1 & 1 & 2 \\
\hline
\end{tabular}


ISSN: 2710-0499 ISSN-L: 2710-0480

\begin{tabular}{lllll} 
& $\%$ & $0,6 \%$ & $0,9 \%$ & $0,7 \%$ \\
\multirow{2}{*}{ Luz y agua } & $\mathrm{N}^{\mathrm{o}}$ & 143 & 73 & 216 \\
\multirow{2}{*}{ Ninguno } & $\%$ & $81,3 \%$ & $66,4 \%$ & $75,5 \%$ \\
& $\mathrm{~N}^{\mathrm{o}}$ & 4 & 10 & 14 \\
\multirow{2}{*}{ Total } & $\%$ & $2,3 \%$ & $9,1 \%$ & $4,9 \%$ \\
& $\mathrm{~N}^{\mathrm{o}}$ & 176 & 110 & 286 \\
\hline
\end{tabular}

Fuente: Encuesta realizada a los beneficiarios del Programa Pensión 65, 2019.

Ahora bien, si entendemos al bienestar social como "el proceso de construcción de mejores condiciones de vida, tanto en lo material como en lo espiritual” (Maguiña, 1988, p. 2), el acceso a los servicios básicos de calidad contribuye al bienestar social de los beneficiarios del programa; por otra parte, desde el enfoque social del bienestar social las necesidades de las personas son cubiertas por el Estado de bienestar, sin embargo, las políticas del Estado de bienestar dejan un amplio campo de necesidades no cubiertas, que hace que el bienestar social no dependa exclusivamente de los niveles económicos de los individuos o de la sociedad en su conjunto, ni de las ayudas que los gobiernos puedan prestar para cubrir las necesidades básicas (Pena, 2009).

\section{Acceso a servicios públicos y bienestar social}

El Programa Pensión 65 promueve la mejora de los mecanismos de acceso de los adultos mayores a los servicios públicos mediante la articulación intersectorial, tanto en educación, salud, y derechos. Los servicios públicos son actividades o acciones que realizan las instituciones públicas en beneficio de las personas adultas mayores. En efecto, el acceso a los servicios públicos es una de las obligaciones del Estado; es así, que las entidades públicas tienen el deber de atender las demandas de los ciudadanos y más aún de los adultos mayores.

Ahora bien, la frecuencia de recurrencia a entidades públicas por grupo de edad de los beneficiarios del Programa Pensión 65, se presenta de la siguiente manera, el 52.8\% del total de beneficiarios encuestados recurren de manera frecuente a los establecimientos de salud; por otro lado, la recurrencia a las municipalidades distritales ocupa el segundo lugar con un $39.2 \%$ del total de encuestados. Sin embargo, respecto a la recurrencia a las entidades públicas por grupo de edad, el $62.8 \%$ de adultos mayores que acuden a los establecimientos de salud tienen las edades de 75 a 79 años respectivamente; véase (Tabla 2).

Tabla 2. Frecuencia de recurrencia a entidades públicas de los beneficiarios del

Programa Pensión 65 por grupo de edad, en los distritos de Puno y Chucuito, 2019

\begin{tabular}{|c|c|c|c|c|c|c|c|c|c|c|c|}
\hline \multirow{5}{*}{ Entidad pública } & \multicolumn{10}{|c|}{ Distrito } & \multirow{3}{*}{ Total } \\
\hline & \multirow{2}{*}{\multicolumn{3}{|c|}{$\begin{array}{l}\text { Puno } \\
\text { Grupo de edad }\end{array}$}} & \multirow{2}{*}{\multicolumn{5}{|c|}{$\begin{array}{l}\text { Chucuito } \\
\text { Grupo de edad }\end{array}$}} & & & \\
\hline & & & & & & & & & & & \\
\hline & 8 & $\frac{\pi}{N}$ & 2 & চ & డ్షి & gి & $\frac{T}{N}$ & 2 & ङ & $\stackrel{\mathscr{G}}{\mathbb{Z}}$ & \\
\hline & in & '́ & 's & $\begin{array}{l}1 \\
\infty\end{array}$ & $\begin{array}{l}\tilde{\sigma} \\
\infty\end{array}$ & î & 'i & is & $\begin{array}{l}1 \\
\infty\end{array}$ & $\underset{\infty}{\tilde{L}}$ & \\
\hline
\end{tabular}

Revista Revoluciones -48- Vol. 1, No 1 (2019), pp. 43-55 


\begin{tabular}{|c|c|c|c|c|c|c|c|c|c|c|c|c|}
\hline & $\mathrm{N}^{\mathrm{O}}$ & 11 & 13 & 22 & 23 & 10 & 20 & 8 & 2 & 3 & $\mathrm{O}$ & 112 \\
\hline Municipio & $\%$ & $\begin{array}{l}50.0 \\
\%\end{array}$ & $\begin{array}{l}34.2 \\
\%\end{array}$ & $\begin{array}{l}37 \cdot 3 \\
\%\end{array}$ & $\begin{array}{l}63.9 \\
\%\end{array}$ & $\begin{array}{l}47.6 \\
\%\end{array}$ & $\begin{array}{l}39.2 \\
\%\end{array}$ & $\begin{array}{l}34.8 \\
\%\end{array}$ & $\begin{array}{l}10.5 \\
\%\end{array}$ & $\begin{array}{l}25.0 \\
\%\end{array}$ & $0.0 \%$ & $39.2 \%$ \\
\hline \multirow{2}{*}{$\begin{array}{l}\text { Establecimientos } \\
\text { de salud }\end{array}$} & $\mathrm{N}^{\mathrm{o}}$ & 11 & 25 & 36 & 12 & 11 & 24 & 13 & 13 & 5 & 1 & 151 \\
\hline & $\%$ & $\begin{array}{l}50.0 \\
\%\end{array}$ & 65.8 & $\begin{array}{l}61.0 \\
\%\end{array}$ & $\begin{array}{l}33 \cdot 3 \\
\%\end{array}$ & $\begin{array}{l}52.4 \\
\%\end{array}$ & $\begin{array}{l}47.0 \\
\%\end{array}$ & $\begin{array}{l}56.5 \\
\%\end{array}$ & $\begin{array}{l}68.4 \\
\%\end{array}$ & \multicolumn{2}{|c|}{$\begin{array}{c}41.7 \% 20.0 \\
\%\end{array}$} & $52.8 \%$ \\
\hline \multirow[b]{2}{*}{ Subprefectura } & $\mathrm{N}^{\mathrm{O}}$ & $\mathrm{O}$ & $\mathrm{O}$ & 1 & 1 & $\mathrm{O}$ & 3 & 1 & O & 2 & 1 & 9 \\
\hline & $\%$ & $0.0 \%$ & $0.0 \%$ & $1.7 \%$ & $2.8 \%$ & $0.0 \%$ & $5.9 \%$ & $4 \cdot 3 \%$ & $0.0 \%$ & $\begin{array}{l}16.6 \\
\%\end{array}$ & $\begin{array}{l}20.0 \\
\%\end{array}$ & $3.1 \%$ \\
\hline \multirow[b]{2}{*}{ Tambo } & $\mathrm{N}^{\mathrm{o}}$ & $\mathrm{O}$ & $\mathrm{O}$ & $\mathrm{O}$ & $\mathrm{O}$ & $\mathrm{O}$ & 3 & 1 & 4 & 2 & 3 & 13 \\
\hline & $\%$ & $0.0 \%$ & $0.0 \%$ & $0.0 \%$ & $0.0 \%$ & $0.0 \%$ & $5.9 \%$ & $4.3 \%$ & $\begin{array}{l}21.0 \\
\%\end{array}$ & $\begin{array}{l}16.6 \\
\%\end{array}$ & $\begin{array}{l}60.0 \\
\%\end{array}$ & $4.5 \%$ \\
\hline \multirow{2}{*}{ Juzgados de paz } & $\mathrm{N}^{\mathrm{o}}$ & $\mathrm{O}$ & $\mathrm{O}$ & $\mathrm{O}$ & $\mathrm{O}$ & $\mathrm{O}$ & 1 & $\mathrm{O}$ & $\mathrm{O}$ & $\mathrm{O}$ & $\mathrm{O}$ & 1 \\
\hline & $\%$ & $0.0 \%$ & $0.0 \%$ & $0.0 \%$ & $0.0 \%$ & $0.0 \%$ & $1.9 \%$ & $0.0 \%$ & $0.0 \%$ & $0.0 \%$ & $0.0 \%$ & $0.3 \%$ \\
\hline Total & $\begin{array}{l}\mathrm{N}^{\circ} \\
\%\end{array}$ & $\begin{array}{l}22 \\
100 \% \\
\end{array}$ & $\begin{array}{l}38 \\
100 \% \\
\end{array}$ & $\begin{array}{l}59 \\
100 \% \\
\end{array}$ & $\begin{array}{l}36 \\
100 \%\end{array}$ & $\begin{array}{l}21 \\
100 \%\end{array}$ & $\begin{array}{l}51 \\
100 \%\end{array}$ & $\begin{array}{l}23 \\
100 \%\end{array}$ & $\begin{array}{l}19 \\
100 \%\end{array}$ & $\begin{array}{l}12 \\
100 \%\end{array}$ & $\begin{array}{l}5 \\
6100 \% \\
\end{array}$ & $\begin{array}{l}286 \\
100 \%\end{array}$ \\
\hline
\end{tabular}

Fuente: Encuesta realizada a los beneficiarios del Programa Pensión 65, 2019.

Como se ha podido apreciar antes, la entidad pública más concurrida son los establecimientos de salud; es así, que también se ha considerado la existencia de estas entidades en ambos distritos, en donde en el distrito de Puno el 69.3\% afirman que si existe establecimientos de salud en sus lugares de residencia; sin embargo, en el distrito de Chucuito el 60\% afirman la ausencia de establecimientos de salud en sus lugares de residencia. Por tanto, la existencia de más establecimientos de salud se presenta de manera limitada en el distrito de Chucuito, considerando que dicho distrito sólo cuenta con cinco establecimientos de salud a nivel de toda la jurisdicción; asimismo, la población de los distritos de Puno y Chucuito manifiesta que acuden a estos establecimientos para la revisión médica correspondiente, control de enfermedades, reducción de la anemia, control de peso y talla respectivamente; al respecto Garay y Román (2016) mencionan que "el derecho a servicios de salud es otro elemento asociado con la seguridad social de los adultos mayores” (p.100).

Por otro lado, los adultos mayores al encontrarse en vulnerabilidad están propensos a contraer enfermedades; es así, que se presentan los tipos de enfermedades que prevalecen en los beneficiarios del Programa Pensión 65 en los distritos de Puno y Chucuito, en este sentido, tenemos a un $24.4 \%$ de beneficiarios del distrito de Puno, que generalmente padecen de artritis y artrosis, seguido de $\mathbf{2 2 . 2 \%}$ de adultos mayores que padecen de problemas auditivos $\mathrm{y}$ visuales, mientras que el $\mathbf{1 4 . 2 \%}$ de beneficiarios no padecen ningún tipo de enfermedad, 
asimismo, el 14.8\% de beneficiarios sufren de hipertensión arterial y por último el 5.7\% de beneficiarios padecen de osteoporosis.

Ahora en el distrito de Chucuito, de igual manera el 30\% de beneficiarios menciona padecer de enfermedades como la artritis y artrosis, seguido de enfermedades producidas por los resfríos con un $28.2 \%$, por último, el $10 \%$ de beneficiarios encuestados de este distrito mencionan tener problemas auditivos y visuales. La existencia de enfermedades en los adultos mayores, se debe a su fragilidad y poca actividad que realizan; al respecto Rodríguez (2006) menciona que el comportamiento sedentario es la ausencia de actividad física, lo que incrementa el riesgo de contraer diversas enfermedades; véase (Figura 1).

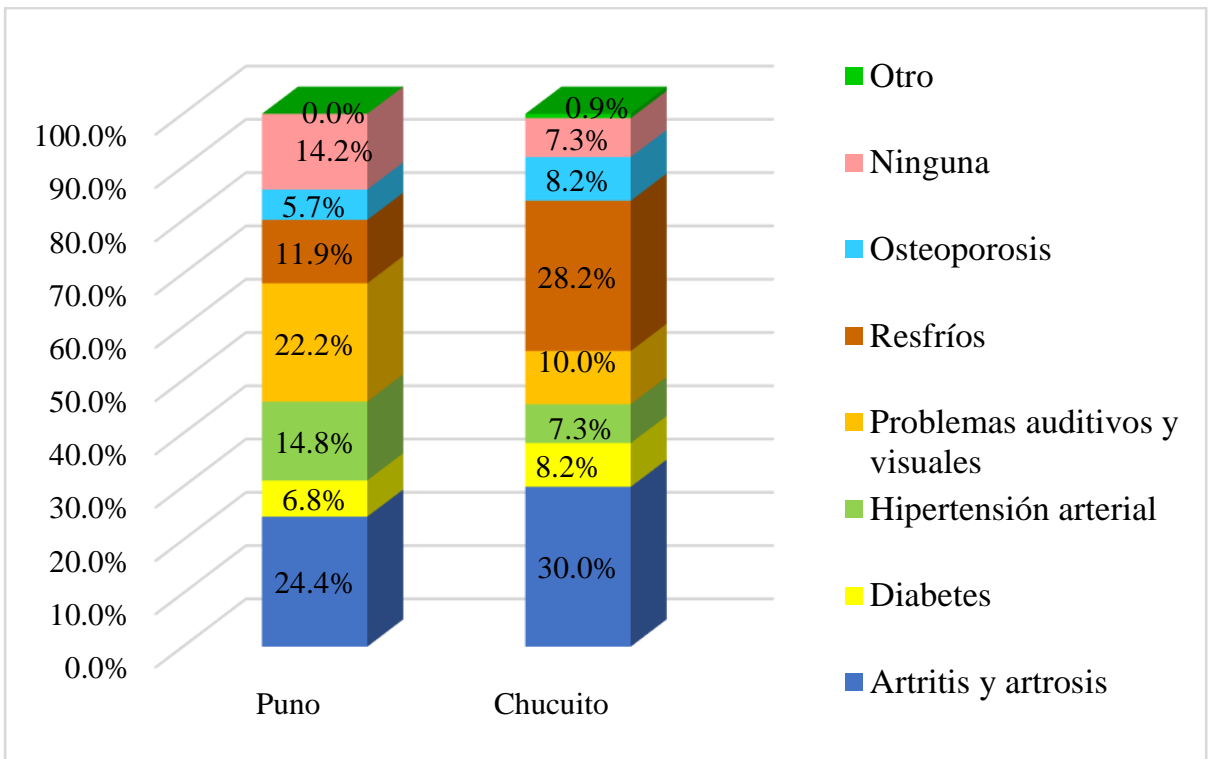

Figura 1. Enfermedades prevalentes en los adultos mayores beneficiarios del Programa Pensión 65 en los distritos de Puno y Chucuito, 2019.

Asimismo, es importante mencionar que respecto al tipo de seguro de salud de los adultos mayores beneficiarios del Programa Pensión 65, el 99.4\% de encuestados del distrito de Puno mencionan tener el Seguro Integral de Salud, y el 98.2\% del total de beneficiarios encuestado en el distrito de Chucuito también afirman contar con dicho seguro de salud; esto nos demuestra, que casi el total de beneficiarios encuestados cuentan con seguro de salud, frente a un (0.6 \% y 1.8\%) de beneficiarios que no tienen ningún tipo de seguro de salud.

Otro de los aspectos que se ha tomado en cuenta en la investigación, fue la frecuencia de realización de actividades físicas por los adultos mayores, en donde el $55.1 \%$ de beneficiarios pertenecientes al distrito de Puno mencionan que realizan actividades que les ayudan físicamente sólo a veces, de igual manera en el distrito de Chucuito, el $49.1 \%$ de beneficiarios encuestado afirman realizar actividades físicas diarias de manera regular; mientras que el $33.5 \%$ y $45.5 \%$ de beneficiarios de los distritos de Puno y Chucuito, mencionan que si realizan actividades diarias que le ayudan físicamente a mantenerse sanos y saludables. Tenemos que considerar que la realización de actividades físicas ayuda en la salud del adulto mayor y en su condición física, para el desarrollo de sus actividades cotidianas; en términos de Rodríguez 
(2006) señala que la actividad física y recreativa programada y sistemática en adultos mayores, contribuye a la mejoría sustancial del bienestar físico, mental y emocional del adulto mayor; relacionarse y compartir jornadas de ejercicio, permite superar problemas de índole social y emocional, además de ello menciona que el ejercicio es una fuente importante de salud y de mejoramiento de las cualidades orgánicas en los adultos mayores.

\section{Saberes productivos y bienestar social}

Saberes productivos es una intervención del Programa Nacional de Asistencia Solidaria Pensión 65, cuya finalidad es promover la protección social de las personas adultas mayores, quienes se encuentran en vulnerabilidad social. La intervención, se implementa en alianza con los gobiernos locales para revalorar a los adultos mayores y su rol como portadores de saberes que refuerzan la memoria colectiva y transmiten a las nuevas generaciones; constituyéndose en un valor para sus comunidades, además, saberes productivos promueve la sistematización de los conocimientos que portan los adultos mayores, con el propósito de transmitirlos a las nuevas generaciones contribuyendo al fomento del sentido de pertenencia y orgullo por la identidad local (Ministerio de Desarrollo e Inclusión Social [MIDIS], 2016).

En esa perspectiva, se ha considerado la frecuencia de participación en dichos talleres y el fortalecimiento de sus habilidades sociales, en donde el $75.5 \%$ de adultos mayores que participan activamente en dichos talleres, ha podido fortalecer su autoestima, ahora los que participan sólo a veces, de igual manera el $22.4 \%$ ha podido fortalecer algún tipo de habilidad social (autoestima, autosuficiencia, comunicación asertiva y empatía); sin embargo, de los que no participan de estos talleres, el $2.1 \%$ no ha podido fortalecer ningún tipo de habilidad social en dichos talleres de saberes productivos.

Asimismo, en los talleres de saberes productivos también se desarrollan actividades físicas, que tiene muchos beneficios, entre ellos: aumentar la longevidad, mejora la capacidad mental, previene y reduce los riegos de enfermedades y mejora la respuesta social, al facilitar una mayor participación en la comunidad y el mantenimiento de redes sociales y vínculos intergeneracionales. (Organización Mundial de la Salud [OMS], 2015). Al respecto, Rubio et al. (2015) mencionan que "la calidad de vida en la vejez tiene que ver con la seguridad económica y con la inclusión social (...), Todo ello promoverá la participación de las personas de edad como miembros activos de la comunidad, una de cuyas funciones puede ser transmitir sus experiencias a las generaciones más jóvenes” (p.2). Además, Rodríguez (2006) menciona que, en cuanto a la situación social del adulto mayor, el hecho de reunirse con las demás personas que integran su grupo, hace que encuentren más amistades y se identifiquen como una gran familia; véase (Tabla 3).

Tabla 3. Participación en los talleres de saberes productivos y habilidades sociales fortalecidas por los beneficiarios del Programa Pensión 65, 2019

\begin{tabular}{lll} 
Distrito & & Total \\
\hline Puno & Chucuito
\end{tabular}




\begin{tabular}{|c|c|c|c|c|c|c|c|c|c|c|c|c|}
\hline \multirow[b]{2}{*}{$\begin{array}{l}\text { Participación } \\
\text { talleres de sal } \\
\text { productivos }\end{array}$} & \multicolumn{4}{|c|}{$\begin{array}{l}\text { Habilidades } \\
\text { fortalecidas }\end{array}$} & \multicolumn{5}{|c|}{$\begin{array}{c}\text { socialesHabilidades } \\
\text { fortalecidas }\end{array}$} & \multicolumn{3}{|c|}{ sociales } \\
\hline & $\begin{array}{r}\text { en } \\
\text { iberes }\end{array}$ & 菉 & 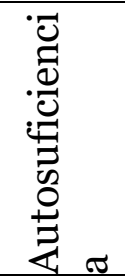 & 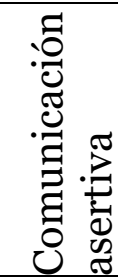 & 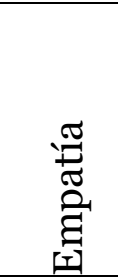 & 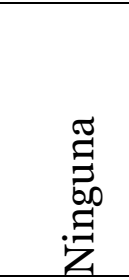 & 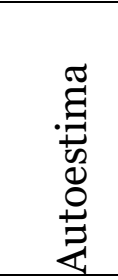 & 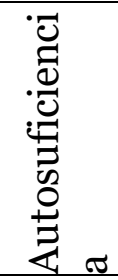 & 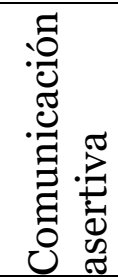 & 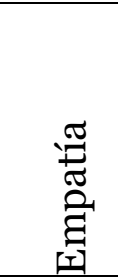 & 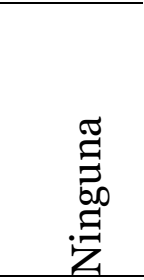 & \\
\hline & $\mathrm{N}^{\mathrm{O}}$ & 0 & 0 & 1 & 0 & 2 & 1 & 0 & $\mathrm{O}$ & 0 & 2 & 6 \\
\hline No participa & $\%$ & $0.0 \%$ & $0.0 \%$ & $2.6 \%$ & $0.0 \%$ & $5.4 \%$ & $1.3 \%$ & $0.0 \%$ & $0.0 \%$ & $0.0 \%$ & $11.8 \%$ & $2.1 \%$ \\
\hline $\begin{array}{l}\text { A veces } \\
\text { participa }\end{array}$ & $\mathrm{S}_{\%}^{\mathrm{N}^{\mathrm{o}}}$ & $\begin{array}{l}19 \\
30.2 \\
\%\end{array}$ & $\begin{array}{l}5 \\
16.1 \%\end{array}$ & $\begin{array}{l}3 \\
7.7 \%\end{array}$ & $\begin{array}{l}2 \\
33 \cdot 3 \\
\%\end{array}$ & $\begin{array}{l}7 \\
18.9 \%\end{array}$ & $\begin{array}{l}23 \\
629.5 \\
\%\end{array}$ & $\begin{array}{l}0 \\
0.0 \%\end{array}$ & $\begin{array}{l}0 \\
0.0 \%\end{array}$ & $\begin{array}{l}0 \\
0.0 \%\end{array}$ & $\begin{array}{l}5 \\
29.4 \\
\%\end{array}$ & $\begin{array}{l}64 \\
22.4 \%\end{array}$ \\
\hline Si participa & $\begin{array}{l}\mathrm{N}^{\circ} \\
\%\end{array}$ & $\begin{array}{l}44 \\
69.8 \\
\% \\
\end{array}$ & $\begin{array}{l}26 \\
83 \cdot 9 \\
\% \\
\end{array}$ & $\begin{array}{l}35 \\
89.7 \\
\% \\
\end{array}$ & $\begin{array}{l}4 \\
66.7 \\
\% \\
\end{array}$ & $\begin{array}{l}28 \\
75 \cdot 7 \%\end{array}$ & $\begin{array}{l}54 \\
69.2 \\
\% \\
\end{array}$ & $\begin{array}{l}5 \\
100 \%\end{array}$ & $\begin{array}{l}5 \\
100 \%\end{array}$ & $\begin{array}{l}5 \\
100 \%\end{array}$ & $\begin{array}{l}10 \\
58.8 \\
\% \\
\end{array}$ & $\begin{array}{l}216 \\
75 \cdot 5 \%\end{array}$ \\
\hline Total & $\begin{array}{l}\mathrm{N}^{\circ} \\
\%\end{array}$ & $\begin{array}{l}63 \\
100 \% \\
\end{array}$ & $\begin{array}{l}31 \\
100 \% \\
\end{array}$ & $\begin{array}{l}39 \\
100 \%\end{array}$ & $\begin{array}{l}6 \\
100 \% \\
\end{array}$ & $\begin{array}{l}37 \\
100 \% \\
\end{array}$ & $\begin{array}{l}78 \\
100 \% \\
\end{array}$ & $\begin{array}{l}5 \\
100 \% \\
\end{array}$ & $\begin{array}{l}5 \\
100 \% \\
\end{array}$ & $\begin{array}{l}5 \\
100 \% \\
\end{array}$ & $\begin{array}{l}17 \\
6100 \% \\
\end{array}$ & $\begin{array}{l}286 \\
100 \% \\
\end{array}$ \\
\hline
\end{tabular}

Fuente: Encuesta realizada a los beneficiarios del Programa Pensión 65, 2019.

Asimismo, se ha tomado en cuenta el nivel de valoración de la lengua materna en la realización de los talleres de saberes productivos y transmisión de saberes ancestrales, en donde en el distrito de Puno el $67 \%$ consideran que es importante el uso de la lengua materna en dichas intervenciones, asimismo el $74.5 \%$ de beneficiarios del distrito de Chucuito, también considera que la lengua materna (aimara) es importante en el desarrollo de saberes productivos; si bien es cierto, el Estado peruano "reconoce y protege la pluralidad étnica y cultural" (Const., 1993, art.19).

Al mismo tiempo, en cuanto a la transmisión de saberes ancestrales a las nuevas generaciones, el $26.7 \%$ y $43.6 \%$ de beneficiarios de los distritos de Puno y Chucuito realizan la transmisión de sus saberes ancestrales de manera oral, mientras que el $34.7 \%$ y $35.5 \%$ de beneficiarios en los distritos de Puno y Chucuito realizan transmisión de saberes ancestrales de manera práctica. Sin embargo, el $38.1 \%$ en el distrito de Puno y el $20.9 \%$ en el distrito de Chucuito no realizan transmisión de saberes ancestrales a las nuevas generaciones; véase (Figura 2). 


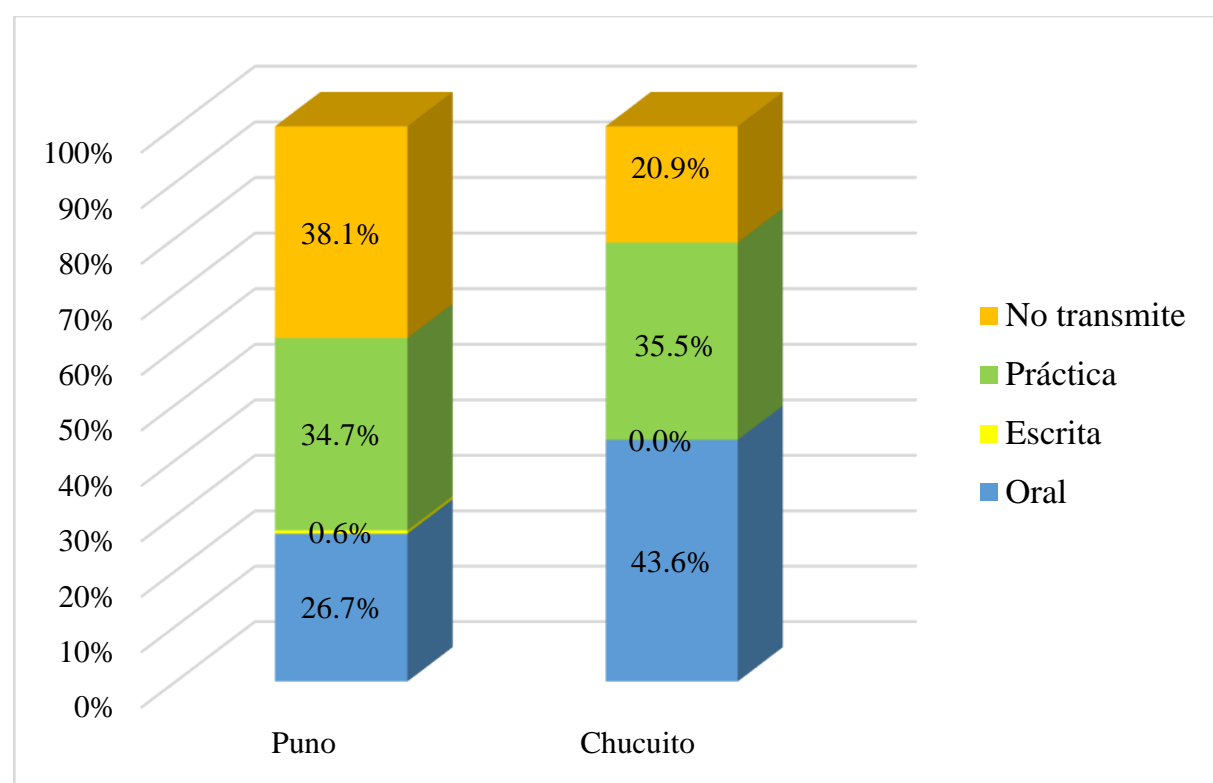

Figura 2. Transmisión de saberes ancestrales a las nuevas generaciones, 2019.

Por último, se ha considerado la convivencia familiar y relaciones sociales de los adultos mayores beneficiarios del Programa Pensión 65 en Puno y Chucuito. Respecto a la convivencia familiar, el 33.5\% de beneficiarios del distrito de Puno mencionan que viven solos y el 24.5\% respectivamente en el distrito de Chucuito también afirman que viven solos. Por otro lado, el $47.3 \%$ de beneficiarios pertenecientes al distrito de Chucuito viven con su cónyuge, y de la misma manera en el distrito de Puno sólo el 26.7\% del total de encuestados mencionan vivir con su cónyuge, como se ha podido apreciar existe abandono familiar en ambos distritos. En ese contexto Molina (2008) citado por Bastos (2009) menciona que "aunque la situación actual de la salud de los adultos mayores haya sido bastante mejorada gracias a los avances de la seguridad social y de la medicina, muchos de ellos aún son sometidos al abandono por parte de la sociedad y de su propia familia" (p.31); véase (Figura $3)$.

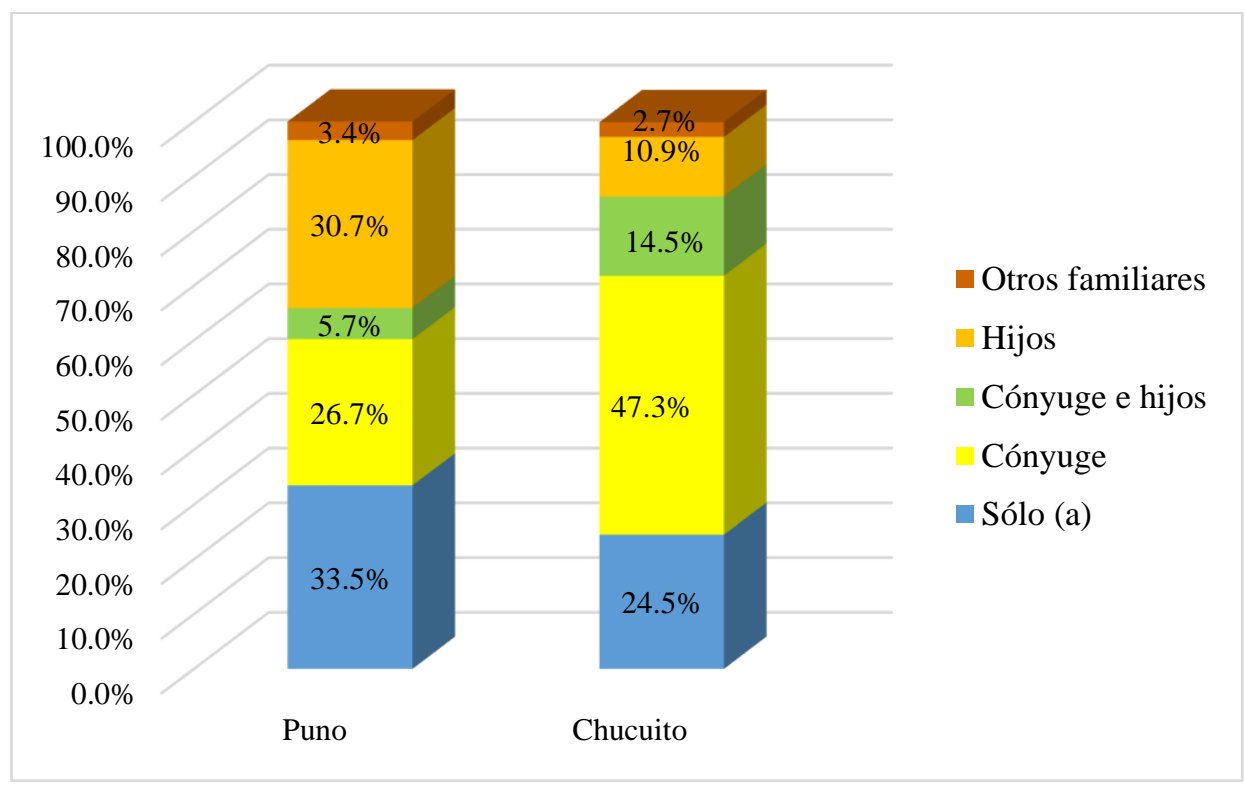


Figura 3. Conformación del hogar de los beneficiarios del Programa Pensión 65 en los distritos de Puno y Chucuito, 2019.

Asimismo, en cuanto a las relaciones sociales de los adultos mayores, se ha podido identificar el tipo de relación que tienes con los demás beneficiarios del programa en su localidad. En ese sentido, el $48.9 \%$ y el $45.5 \%$ de beneficiarios de los distritos de Puno y Chucuito mencionan tener una relación muy buena con los demás beneficiarios de su localidad, mientras que el $39.8 \%$ y el $32.7 \%$ asumen tener una relación adecuada con los demás beneficiarios. Sin embargo, solo el $0.6 \%$ y $0.9 \%$ de beneficiarios en ambos distritos afirman tener una relación mala y muy mala a la vez con los demás beneficiarios del Programa Nacional de Asistencia Solidaria Pensión 65.

Es necesario subrayar, que la comunicación y relación con los demás actores sociales es importante para mantener el proceso interacción social mediante la socialización en la sociedad; al respecto Mead (1920) citado por Ritzer (1993) menciona que "el pensamiento se configura y refina mediante el proceso de interacción social centrada en la socialización (...), y en todo este proceso las personas no solo aprenden a vivir en sociedad (adquirir un rol, la cultura, etc.) sino que más bien desarrollan su capacidad de pensar de una manera distintivamente humana a través de un proceso dinámico y adaptativo" (p.238). En efecto, es necesario que haya interacción entre las personas adultas mayores para que estos actúen teniendo en mente a los demás.

\section{CONCLUSIONES}

La subvención económica otorgada por el Programa Pensión 65 no tiene relación significativa con el bienestar social de los beneficiarios en los distritos de Puno y Chucuito; además de ello el dinero destinado en su mayoría es destinado para cubrir necesidades de alimentación, gastos de salud, vestimenta y transporte, por lo que es limitado, Sin embargo, la subvención económica brinda cierto grado de seguridad económica, por ser la única fuente de ingresos de gran parte de los beneficiarios en ambos distritos.

El acceso a los servicios públicos por parte de los beneficiarios del Programa Pensión 65 es independiente al bienestar social de los beneficiarios en los distritos de Puno y Chucuito; por tanto, no hay una relación significativa, ya que los beneficiarios tienen limitaciones al momento de concurrir a una institución pública, situación que se encuentra condicionada por la limitada existencia de establecimientos de salud, y tenencia de enfermedades por parte de los adultos mayores.

La intervención de saberes productivos tiene una relación significativa con el bienestar social de los beneficiarios del Programa Pensión 65 en los distritos de Puno y Chucuito, porque a partir de la intervención de saberes productivos se promueve la participación activa de los adultos mayores, y se fortalecen las habilidades sociales; tomando en cuenta el uso de la 
lengua materna en el desarrollo de las intervenciones, además de fomentar la transmisión de saberes ancestrales a las nuevas generaciones.

\section{REFERENCIAS BIBLIOGRÁFICAS}

Ambrosio, F. L., Cisneros, L., y Condezo, T. K. (2017). Impacto del Programa Nacional de Asistencia Solidaria Pensión 65 en el bienestar social de los adultos mayores en el distrito de Huácar - Ambo, Huánuco, 2016 (tesis de pregrado). Universidad Nacional Hermilio Valdizán, Huánuco, Perú.

Bastos, A. (2009). Mejorando la Calidad de Servicios para el Adulto Mayor: una Aproximación Constructivista (tesis de maestría). Universidad de Chile, Santiago, Chile.

Blum, V. (2005). Campesinos y teóricos agrarios. Lima, Perú: Instituto de Estudios Peruanos.

CEPAL - CELADE. (2007). Ministerio de Bienestar Social de Ecuador. Estudio sobre la protección social de la tercera edad en Ecuador.

Constitución Política del Perú [Const.] (1993). Articulo 19 [Título I]. 1ra Ed.

Garay, S., y Román, A. (2016). Condiciones de vida de las personas mayores beneficiarias del programa de apoyo al adulto mayor en Nuevo León. Iberóforum revista de Ciencias Sociales, $\quad 11 \quad$ (22), $\quad 98-116 . \quad$ Recuperado de http://www.redalyc.org/articulo.oa?id=211049386004

Gonzáles, L. B., y Julca, F. G. (2018). Programa "Pensión 65" y la Calidad de Vida de los adultos mayores del Distrito de Huancas, Amazonas (tesis de maestría). Universidad Cesar Vallejo, Chiclayo, Perú.

Instituto Nacional de Estadística e Informática. (2018). Adultos mayores de 70 y más años de edad, que viven solos. Recuperado de https://www.inei.gob.pe

Instituto Nacional de Estadística e Informática. (2018). Puno: resultados definitivos de los Censos Nacionales 2017: XII de Población, VII de Vivienda y III de Comunidades Indígenas.

Recuperado de http://www.inei.gob.pe/media/MenuRecursivo/publicaciones_digitales/Est/lib1563/

Instituto Nacional de Estadística e Informática. (2018). Situación de la Población Adulta Mayor. (02). Recuperado de https://www.inei.gob.pe/media/MenuRecursivo/boletines/o1-informe-tecnicono2_adulto_ene-feb_mar2018.pdf

Maguiña, A. (1988). Bienestar social y Trabajo Social Alternativo. Revista de Acción Crítica, 1 (23), 1-6. Recuperado de http://www.ts.ucr.ac.cr/binarios/accioncritica/ac-cr-02304.pdf

Ministerio de Desarrollo e Inclusión Social. (2016). Saberes productivos: guía para los equipos designados por los gobiernos locales. Recuperado de https://www.pension65.gob.pe/wp-content/uploads/2018/o6/GUIA-SABERES.pdf Organización Mundial de la Salud. (2015). Informe Mundial sobre el Envejecimiento y la Salud. Recuperado https://apps.who.int/iris/bitstream/handle/10665/186466/9789240694873_spa.pdf? sequence $=1$ 
Pena, B. (2009). La medición del Bienestar Social: una revisión crítica. Estudios de Economía $\begin{array}{lllll}\text { Aplicada, } & 27 & \text { (2), } & \text { 299-324. }\end{array}$ http://www.redalyc.org/articulo.oa?id=30117056001

Ritzer, G. (1993). Teoría sociológica contemporánea. 3ra ed. México: McGraw-Hill.

Rodríguez, M. (2006). El ejercicio físico y la calidad de vida en los adultos mayores. Revista Pensamiento actual, 6 (7), 134-142. Recuperado el 05-06-2019 de https://dialnet.unirioja.es/descarga/articulo/5897940.pdf

Rubio, D. Y., Rivera, L., y Borges. (2015). Calidad de vida en el adulto mayor. Varona, (61), 1-7. Recuperado de http://www.redalyc.org/articulo.oa?id=360643422019

Salvador, M. L. (2015). Influencia del Programa Nacional de Asistencia Solidaria Pensión 65 en las condiciones de vida de los de los beneficiarios del distrito de Quiruvilca. Provincia de Santiago de Chuco. Departamento La libertad, año 2014 (tesis de pregrado). Universidad Nacional de Trujillo, Perú. 